to doubling the coefficient of skin friction. If the aerofoil were in the first instance designed for least resist ance, the author has shown that the skin-frictional re sistance will be just half the total resistance; hence unless we readjust the design to suit the new condtions the return stroke uses half as much energy as the working stroke, and the efficiency will be 66.6 per cent It is evident, however, that for best efficiency the wing area must be altere to continue to comply with the conditions of least resistance, and the new design will be given by employing a coefficient of skin friction double of its actual value. Theory shows that this will give a resistance $\vee 2$ times greater than previous$1 y$, approximately in the relation 10:7; thus the effciency will be about 70 per cent.

Now, in the case of wing motion the angular char acter of the movement renders the problem more complicated, but the author will venture the suggestion that whatever in actual wing motion does not partake of the action of a screw propeller is accounted for as above, and that which does partake of the screw propeller is subject to the loss of efficiency inherent to the screw propeller in its simplicity; hence the efficiency of wing propulsion will lie between the value above ob tained and that proper to a well-formed screw propeller.

Now, it is a fact that serves our present purpose exceedingly well that, so far as we have knowledge the best efficiency obtainable with the screw propeller is approximately the same as obtained above for the reciprocating aerofoil; hence there is every reason to believe that wing propulsion has an efficiency of approximately 70 per cent, and thus possesses no ad approximately 70 per cent, and thus possesses no ad-
vantage on the score of efficiency over.a well-designed screw.

The possibility or otherwise from a physiologica point of view of a bird being able to generate suffi-

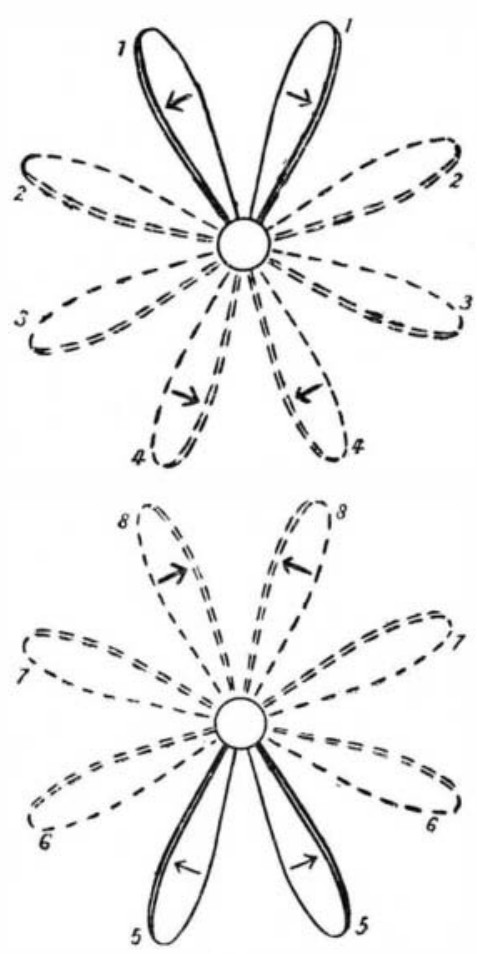

Fig. 23.

cient power for flight is a ground on which estimates of power expenditure have sometimes been challenged. It must be remembered that birds of over about 1 pound weight have, generally speaking, a very limited capacity for active flight; they principally employ the soaring mode. It must also be remembere that the soaring mode. It must also be remembered that the
power varies as $L^{2}$ law applies very closely to animal power varies as $L^{2}$ law applies very closely to animal
life as well as to machines, and that a direct consequence of this law is that the rate at which an animal can increase its altitude is-other things being equalinversely proportional to the cube root of its weight.

Let us take the homing pigeon and the horse for purposes of comparison; although the two, considered as machines, are of widely different type, both have been bred carefully as power producers, and the comparison should yield results of the right order.

Assume, as indicated in the present paper, a gliding angle $=1$ in 6 and a flight velocity of 54 feet per second : that is to say the rate of gliding descent will be $54 / 6=9$ feet per second, or, taking the efficiency of $54 / 6=9$ feet per second, or, taking the efficiency of
wing propulsion as estimated at 70 per cent, this corresponds to an increase of 13 feet per second altitude as representing the work done in flight. Taking the horse as weighing equal to 1,300 pigeons, say $\left(11^{3}\right)$ pigeons, we have the rate at which a horse should be able to increase its altitude $13 / 11$, or 1.18 feet.

There seems nothing improbable in the above result, for a horse of 1,200 pounds weight it is equivalent to slightly over $21 / 4$ horse-power, an output of which such a horse should be capable for quite a considerable time daily. It would thus appear that the limiting size of birds of active habit may be approximately predicted by modern flight theory from the known working capacity of a horse.

There is a variety of active flight practise more particularly by some of the smaller species which the author has termed "leaping" or "bounding" flight. In this mode the bird may be seen to progress through the air by a series of bounds; close observation shows that the "take-off" consists of a period of active flight, and the leap is a simple parabolic trajectory performed

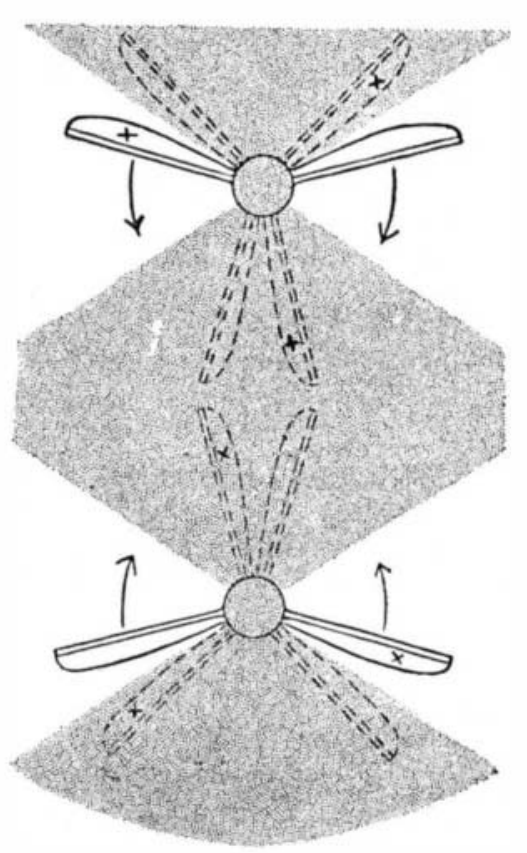

FIG. 24. like a projectile with the wings closed. The author has discussed this mode of flight in his "Aerodynamics"; * it may be accounte for on the grounds that the bird when exceeding its normal velocity of flight -that for which it is designe-endeavors to approximate to the conditions of least resistance by closing its wings intermittently to obviate skin friction. The faster the bird flies the shorter relatively becomes the active period; this is as theory requires. It is worthy of note that apart from body resistance the energy expended for a given distance will be approximately th same, whether the bird is flying steadily at its norma velocity, or whether it be flying at an accelerate veloc ity by the leaping method.

It is only the smaller birds that indulge in the leaping mode of flight; it is a very common mode of progression among the waghtail group; the largest exponent of the leaping mode observed by the author is th green woodpecker. The reason for this limitation i the question of size as affecting power development with which we are already familiar. Thus let us sup pose that a given bird be correctly proportioned for flight at, say, 40 miles per hour, and that its maximum sustained power output is just sufficient for flight at this velocity; then in the case of a geometrically similar bird, the law of corresponding speed will give the velocity for which it is correctly proportioned, and thi velocity for a bird of half the linear size will be 40 divided by $\sqrt{ } 2$, or approximately 29 miles per hour. But such a bird will, at this speed, have an enormous surplus of power at command, for it should be capable of lifting itself at twice the rate of the larger bird, and thus of flying at a far greater velocity; hence, al though adapted by design for a velocity of only 29 miles per hour, it can, by adopting the leaping mode, attain a much higher speed; in fact, on elementary theoretical grounds at least, it should be thus capable of a speed higher than that of the larger bird. The body resistance probably becomes formidable when the body resistance probably becomes formidable when the
smaller bird flies as fast as the larger, and puts a smaller bird flies as fa
limit on this conclusion.

Another field in which the horse-power weight factor is of moment is in the capacity of a bird for stationary

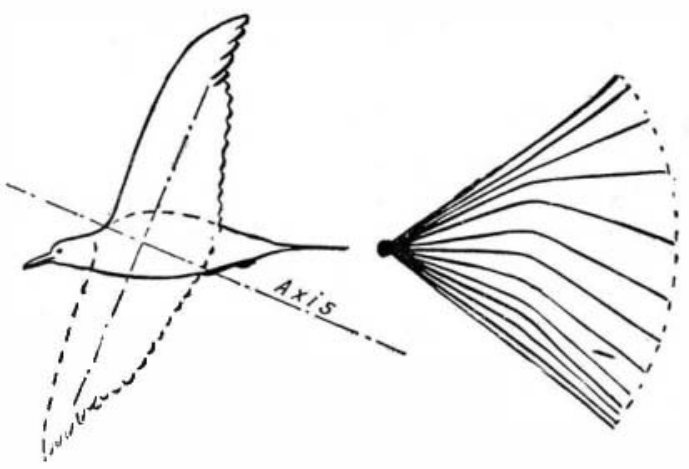

FIG. 25.

FIG. 26.

flight, and hand in hand with this the capacity to rise from the ground without a preliminary run; again the advantage obviously rests with the smaller bird, a con clusion that is fully borne out by observation.

There is one kind of flight that must be admitted to be a vexed question. According to observers having a reputation for accuracy, the migrations of birds are carried out at almost incredible velocities, running sometimes into hundreds of miles per hour. It is quite possible that flights of this kind sometimes occur,' but it would be absurd to suppose that the said velocity is that of flight; it rather represents the velocity of some great aerial current on which the bird is borne, and of which the flight velocity of the bird constitutes but a small and unimportant part. If this conclusion is at fault, the phenomenon of migratory flight must be something quite apart from that of flight under normal conditions. The rarefaction of the air at high altitudes may account for some in. crease in the flight velocity under the conditions of migration, but theory gives the increase on this score as inversely proportional to the square root of the density, an amount which is quite inadequate as an explanation.

\section{THE THEORY OF DYEING.}

By S. H. Hians, M.Sc.

Perhaps there is no part of chemistry concerning which so many speculations have been made, and so few really quantitative experiments carried out, as that portion dealing with the actions taking place during dyeing operations. Many observations have been made and deductions drawn, but yet there is a surprising lack, in this branch of applied chemistry, of the large amount of accurate work which is so characteristic of practically all other branches of chemical science. The art of dyeing is very old, for Pliny observed polygenetic color dyeing and the application of different mordants; nevertheless, we have not progressed very far toward a true explanation of the phenomena observed during the dyeing of textile fibers.

The different theories which have been advanced may be divided into (1) the chemical theory; (2) the solution theory; (3) the mechanical theory. It is true that other theories also exist of the nature of chemico-

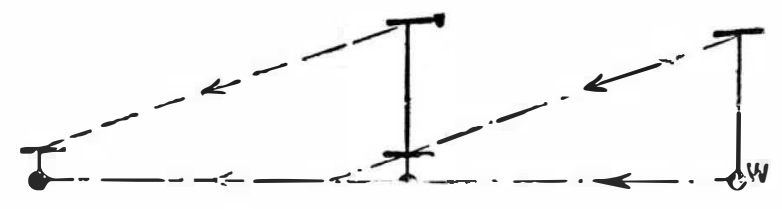

FIG. 27.

mechanical, but the above are the definite classes of opinion. The chemist with pardonable pride attempts to explain the process by saying that chemical combination takes place during dyeing, and that the product is mainly the result of chemical action between the fiber and the dye-stuff. He is imbued with the all-embracing nature of his science, and resembles the apostle of electrolytic dissociation who is busy attributing all chemical actions to the whims of those mysteries-the ions.

It is evident from the literature which is issued from time to time that most investigators of the dyeing process support the chemical theory; but it is also noticeable that most of their conclusions are based

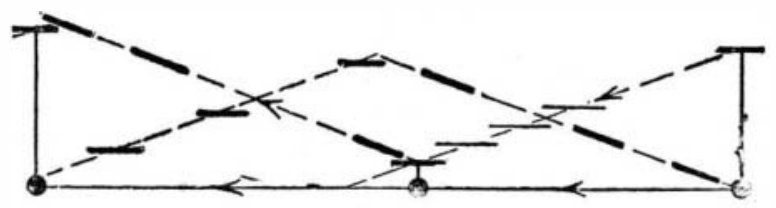

FIG, 28.

upon investigations of animal fibers, as silk and wool, whereas the actions taking place during the dyeing of vegetable fibers, as cotton and linen, have been left almost entirely without elucidation. It is maintained that silk and wool are capable of entering into definite chemical reactions with dye-stuffs, but whereas it is realize that cellulose, which is the chief constituent of vegetable fibers, cannot exhibit strong acidic or basic properties, yet the vegetable kingdom is left alone, and broad generalizations are made from experiments on the other fibers mentioned. At the outset it must be recognized that any theory of dyeing must include all classes of dyeing operations.

The solution theory was advanced in 1890 by Witt, and was the attempted application of the solid solution theory of physical chemistry to explain the phenomena observed during dyeing operations. This theory displayed the genius of its author, and afforded plausible explanations of many experiments, but in other respects it was found lacking. Witt drew attention to the fact that wool dye with magenta was of the same color as a water solution of magenta, and not the dark green color of the magenta crystals. He said that this observation supported his contention that the magenta was dissolved in the wool. It was shown by Von Georgevics, however, that if magenta crystals are only made fine enough they also exhibit the red color of the solution in water. Further, Walker and Appleyard, working with picric acid, made observations which were incomparable with Witt's conclusions, and thus the new theory of dyeing was shaken at its foundation.

The formation of theories is usually left to the greatest minds, to those of a philosophical turn, and it is possible that in this selection long experience 
of practical dyeing has seidom entered into the formulations.

The mechanical theory is to the effect that the dyestuff is drawn from solution and held in some peculiar physical manner by the fiber. Walter Crum gave splendid exposition of this view in the sixties, but since that time chemical theory has been so much in evidence and chemical explanations so plausible that the process of dyeing has had to fall in and submit to the popular explanation. Nevertheless, on reading Crum's work one cannot help being struck with its merit, and one marvels that, in spite of this wellknown work, the chemical theory of dyeing has gained so many adherents.

In support of the mechanical theory of dyeing some papers have recently been issued, e. g., Hïbner, "Experimental Investigation of the Process of Dyeing" (Trans. Chem. Soc., 1907). It is well known that charcoal and other solids possess the property of extracting dye-stuffs from solution; in the case of char-
coal the tinting effect is not noticeable, but if a white substance, as China clay, be used, then it is seen that the solid is dyed. It has been stated that in such cases Huibner showe that these solids behaved either like wool or like cotton during the treatment with water to remove the dye-stuff. Thus it was found that charcoal behave similarly to wool during every treatment to which wool is submitted in commercial dyeing, and likewise graphite was found to fall in with the usual methods of dyeing cotton; in both cases the fastness to washing was similar.

In pursuance of this line of research the present writer has recently issued (Higgins, Journ. Soc. Chem. Ind., 1909, xxviii, 188) an account of experiments which further support the mechanical theory of dyeing. Wool was beaten in a beating engine to five different states of division, and the samples obtained stuff from solution and moisture from the air. It was found that the maximum absorption of dye-stuff and of moisture took place during, approximately, the same time, and that this maximum amount in both cases was practically constant for all states of division. Samples of cotton mercerized, bleached, and un bleached, were found to absorb iodine from weak po tassium iodide solution in proportion as they absorbed moisture from the air. A striking result was obtained by plotting the moisture contents of cotton yarn mercerized with different strengths of caustic soda solution. The moisture curve was found to closely resemble the dye trial curve obtained when a substantive cotton dye-stuff was used; in short, the mercerized cotton samples only absorbed more dye-stuff in so far as they absorbed more moisture. The experiments bring out a marked similarity between the absorption by textile fibers of a solid from a liquid medium, and of a vapor from a gaseous medium, and as the absorption of moisture from air is relegated to that class of little understood phenomena known as surface attraction, it must therefore be taken that the dyeing of textile fibers also belongs to that class of physical action.-Chemical News.

\section{$\begin{array}{lllllllllllllllll}\text { L } & \text { I } & \mathbf{F} & \mathbf{E} & \text { I } & \mathbf{N} & \mathrm{O} & \mathbf{T} & \mathbf{H} & \mathbf{E} & \mathbf{R} & \mathbf{W} & \mathbf{O} & \mathbf{R} & \mathbf{L} & \mathbf{D} & \mathbf{S} .\end{array}$} THE INTERPRETATION OF PLANETARY MARHINGS.

\section{B Y F. W. H E N H E L, B.A., F.R.A.S.}

THE question of the habitability of "other worlds than ours" has always been of great interest to us as inhabitants of this earth, and speculations have at all times been entertained as to the probable nature and mode of existence of extra-terrestrial beings. Fontenelle, an ingenious French writer of some note in the early eighteenth century, composed a series of dialogues between himself and a fashionable lady, "the Marchioness," on the different planets of the solar system, attributing to them inhabitants whose qualities were in accordance with the influences respectively attributed to these bodies in astrological systems. The inhabitants of Mercury were of hot, "mercurial" temperament, those of Venus soft and amorous by nature, those of Mars warlike and belligerent, and so forth. In later times, the famous Dr. Whewell in dulged in some speculations as to the possible physical nature of the inhabitants of the various planets of our system.

However, it must be confessed that little scientific evidence, one way or the other, exists as to such matters, and, indeed, there are many who consider-not altogether without reason on their side-that our earth alone in the universe is the abode of organized earth alone in the universe is the abode of organized
beings. This, no doubt, may seem an extreme view, though it may at once be conceded that it is not easy to imagine how many of the forms of life with which we are conversant on this planet could exist even on the nearest of our neighbors in space. The very variety of conditions under which we know life to be possible here, however, should of itself preclude us from asserting that life can only exist, in our own solar system at least, on this planet. Nothing seems to prevent the existence of totally different beings on every one of the planets (with the possible exception of the moon), organized and just as fitted for the conditions of their existence as we are for our own. As for the "fixed" stars, though their intense heat seems to preclude the present existence of life, yet, as they are in all probability surrounded by revolving planets not unlike those in our own system, there is no doub that on some of these latter conditions prevail more or less akin to those existing here. Where we know nothing we may speculate without fear of contradic tion.

Our own solar system presents a considerable variety of bodies at varying distances from their centra orb, and of very various sizes. The great distances of the planets Jupiter and Saturn from the sun, and the probability that their surfaces are still more or less incandescent, seem to be against the possibility of their being inhabited, though there is no reason whatever, so far as we know, why some of their satellites, at least, should not be the abode of living beings. The planet Mercury, from its proximity to the sun, and from its turning one side only toward the latter, seems at present unfitte for habitation. The case is different, however, with Venus and Mars, our two nearest neighbors in the planetary system. Though much less is known as to the physical conditions of Venus than might be expected from its comparative prox imity, approaching as it does at times within $26,000,000$ miles, a distance less than that of any other heavenly body (the moon only excepted), yet sufficient is known to show a eonsiderable analogy of conditions with those prevailing on our own planet. Its size is slightly less than that of the earth (diameter 7,700 miles, surface and volume correspondingly less than in our planet), and from markings detected on its surface, it has been conclude that it turns round once on its own axis in a period of $23 \mathrm{~h} .21 \mathrm{~m}$., so that its "day" is nearly of the same length as our own, though some have contended for a much longer period. Observers in Italy and other more favore regions have distinctly recognized markings on the planet's surface indicating the existence of continents and oceans "dimly visible," while intensely bright spots; like the polar caps so well seen on Mars, have also been seen at times. Schröter and other observers long ago concluded the existence of high mountains upon the surface. When Venus is near the sun, distinct evidence of the existence of an extensive atmosphere twice as dense as our own is obtained, and the spectroscope shows the presence of water-vapor in some abundance. The dark portion of the planet's disk (that turned away from the sun) is occasionally seen faintly illuminated, in manner, says Prof. Young, recalling the aurora and other electrical manifestations on the earth.

For a long time more or less conflicting evidence as to the existence of a possible satellite or attendant on Venus was given; but it seems fairly certain that if any such body exists it must be a very small object. On the other hand, the absence of a "moon" is to great extent made up for by the earth. It may, perhaps, seem strange to some to think of our own apparently dark earth as a luminary; but there is no doub that, as seen from Venus, if there are any inhabitants, it must be at times a brilliant object. When Venus is nearest to the earth (nearly between earth and sun), its dark side is turned toward us, and, being also i the region of the sky close to the sun, it is invisible to us (except on the rare occasion of a "transit") but, on the other hand, the earth, having its illuminated side turned wholly toward Venus, must be conspicuously visible as a "full" disk of its maximum size, shining during the night, in the sun's absence, with a brilliancy greatly exceeding the maximum brightness of Venus as seen by us. Thus the want of a moon to the planet may be to a great extent made up for in this way.

The distance of Venus from the sun is only about three-quarters that of the earth, or about $67,000,000$ miles, so that any area of its surface must receiv about twice the amount of light and heat that an equal area on the earth receives; but, as we have alread said, the presence of a more extensive atmosphere may to a considerable extent mitigate this, to our idea, excessive amount

"In size, density, and general constitution," say Prof. Young, "this planet is our earth's twin sister." Its diameter is slightly smaller, its period of rotation slightly less (day), while its year is only a little ove seven months. Air, water, lands, continents, moun tains, polar snows, etc., all seem to be present. Thus, so far as our limited knowledge extends, the evidence for the existence of living beings, of a character not so very dissimilar from those with which we are familiar, seems as complete as we can reasonably expect. Turning now to Mars, we meet with a planet ver considerably smaller. Its diameter is only about 4,200 miles, its mass less than one-ninth that of our own earth, and its volume only one-seventh. Its distance from the sun varies from about 135 to 148 millions of miles, and it completes one revolution in 687 days, turning on its axis once in 24 hours 37 minutes. It equator makes an angle with the plane of its orbit of about 25 deg., which is not much greater than the angle between the earth's equator and its orbit; so that, so far as the seasons depend upon this condiion, there should not be much difference in this respect for the two planets. Like our own earth, Mars is not quite spherical, but slightly flattened at the pole, and this flattening is nearly the same in both cases. There are two small satellites, or moons; but they were only discovered in 1877 by the Washington observers with the great 26 -inch refractor. Neither of these bodies exceeds ten miles in diameter, so that they cannot give much light to their primary, or produce any considerable tides. Phobos, the inner of these two satellites, revolves round Mars in less than eight hours-a period much shorter than that in which the planet makes one rotation on its axis. Thus it will appear to rise in the west and set in the east. The outer satellite, Deimos, will rise in the east, like the other heavenly bodies; but its orbital motion in the reverse direction is nearly as great, so that it will take about 130 hours between rising and setting, undergoing all its changes of phase-"new," "full," etc. -four times in that interval, its period of revolution being only thirty hours. Frequent eclipses of these satellites and transits across the sun's disk take place. In a small telescope some markings may usually be detected upon the planet's surface. In a more powerful instrument much detail can be perceived. The planet's disk will be seen to be not so distinctly reddish as it appears to the naked eye, but still of that color, though there will also be found green and purple patches of more or less distinct outlines. Near either pole are to be seen brilliant white spots, which periodically increase and diminish according to their respective presentation away from or toward the sun, the spot at the pole turned away from the sun increasing, the other diminishing, just like the polar snows on our planet, whence the idea naturally arose that these spots on Mars are due to a similar cause. The details of the surface vary considerably from time to time. Spots and markings seen distinctly at one time are scarcely visible at another, while other markings are seen instead (the color of the surface varies at times), in addition, of course, to the change produced by the rotation of the planet. These variations are probably due to the atmosphere of the planet, laden with clouds and mists, obscuring more or less of the underlying features of the planet. By comparison of drawings made from time to time, maps have been made giving the permanent features of the planet's surface. There seems, so far as we can judge, a much greater proportion of land to sea than is the case with the earth, few great continents and oceans, but numerous narrow "seas" penetrating and dividing the "land." But the most remarkable features of this planet's surface are a series of long, straight markings connecting the larger "seas," first seen by Schiaparelli in 1877, to which he gave the name of "canals." In 1881 he saw them again, and this time many of these canals were double. Some of his observations have been confirmed by other astronomers; and others, again, have denied the existence of these canals, or, at least, their duplicity. M. T'erby considers the "double canals" to be purely an optical phenomenon, and remarks that they present exactly the appearance that single markings would present if viewed through a double-image prism. Some experiments made by Mr. Maunder at Greenwich point to a 